\title{
COEDUCACIÓN Y EXPERIENCIAS POSITIVAS. APORTACIONES A LA CONVIVENCIA DESDE LA EXPRESIÓN MOTRIZ COOPERATIVA
}

\author{
COEDUCAÇÃO E EXPERIÊNCIAS POSITIVAS. CONTRIBUIÇÕES PARA A \\ CONVIVÊNCIA A PARTIR DA EXPRESSÃO MOTRIZ COOPERATIVA
}

\author{
CO-EDUCATION AND POSITIVE EXPERIENCES. CONTRIBUTIONS FOR \\ COEXISTENCE BY COOPERATIVE MOTOR EXPRESSION
}

\author{
Unai Sáez de Ocáriz Granja*, Jorge Serna Bardavio **, Pere Lavega Burgués **, \\ Antoni Costes Rodríguez **, Jaume March Llanes **
}

Palabras clave:

Afecto.

Análisis

Cuantitativo.

Placer.

Educación Física.
Resumen: Crear experiencias positivas en Educación Física es uno de los aspectos clave para favorecer la educación de relaciones interpersonales. Este estudio examinó el efecto de las situaciones motrices de expresión cooperativa (SMEC) sobre los estados de ánimo (EA) de universitarios al participar en grupos segregados y mixtos. Participaron 81 alumnos (22 chicas y 59 chicos) del grado en Ciencias de la Actividad Física y el Deporte (INEFC Barcelona). Al inicio y final de cada sesión los participantes cumplimentaron el cuestionario POMS. El análisis estadístico mediante ecuaciones de estimación generalizadas mostró que en ambos grupos se mejoró el EA de los participantes. Los valores de tensión-ansiedad, depresión-abatimiento fueron más favorables en los grupos mixtos, mientras que puntuación global, vigor-actividad, fatigainmovilidad fueron superiores en los grupos segregados. Los hallazgos confirman la contribución de las SMEC para la creación de ambientes positivos y la mejora de la convivencia en y entre ambos géneros.

Palavras chave:

Afeto.

Análise quantitativa.

Prazer.

Educação Física.
Resumo: Criar experiências positivas em Educação Física é um dos aspectos chave para favorecer a educação de relações interpessoais. Este estudo examinou 0 efeito das situações motrizes de expressão cooperativas (SMEC) sobre os estados de ânimo (EA) de universitários ao participarem em grupos separados e mistos. Participaram 81 alunos (22 meninas e 59 meninos) do curso de graduação em Ciências da Atividade Física e Esporte (INEFC Barcelona). Ao início e ao final de cada sessão os participantes preencheram o questionário POMS. A análise estatística mediante equações de estimativa generalizadas mostrou que em ambos os grupos melhorou os EA dos participantes. Os valores de tensãoansiedade e depressão-abatimento foram mais favoráveis nos grupos mistos; enquanto pontuação global, vigor-atividade e fadiga-imobilidade foram superiores para os grupos separados. Os resultados confirmam a contribuição das SMEC para a criação de ambientes positivos e a melhora da convivência em e entre ambos os gêneros.

Abstract: Creating positive experiences in Physical Education is one of the key aspects to promote education for interpersonal relations. This study examined the effects of cooperative expression motor situations (SMEC) on the moods of college students participating in segregated and mixed groups. It included 81 students ( 22 girls and 59 boys) of the Science of Physical Activity and Sport degree (INEFC Barcelona). At the beginning and end of each session, participants completed the POMS questionnaire. Statistical analysis using generalized estimating equations was conducted and showed that participants' moods were improved in both groups. Tension-anxiety, depression-dejection values were more favorable in mixed groups while overall score, vigor-activity, fatigue-immobility values were higher in segregated groups. The findings confirm the contribution of SMEC for creating positive environments and improving coexistence in and between both genders.
*Universidad de Barcelona. Barcelona, España.

E-mail: usaez@gencat.cat

**Universidad de Lleida. Lleida, España. E-mail: jserna@inefc.es; plavega@inefc.es; tcostes@inefc.es; jmarch@pip.udl.cat

Recebido em: 04-04-2017 Aprovado em: 27-07-2017

(c) (1) (8) Licence 


\section{INTRODUCCIÓN}

Las desigualdades en materia de género continúan siendo una realidad en la sociedad actual, especialmente en el contexto escolar, tal y como lo demuestran diferentes informes realizados por instituciones internacionales (UNESCO, 2012). Los agentes que intervienen en el sistema educativo deberían asumir la responsabilidad de transformar y proponer una educación democrática e inclusiva que contribuya a superar las expresiones sexistas que se reproducen generación tras generación (ARNAIZ, 2012, AZORÍN, 2014, GARCÍA-PERALES, 2012, GIL-MADRONA et al., 2014).

Una de las prioridades de la educación actual es la creación de ambientes de bienestar que permitan tanto al profesorado como al alumnado desarrollar en óptimas condiciones los procesos de enseñanza aprendizaje (ÁLVAREZ-GARCÍA et al., 2010, GARCÍA-RAGA; LÓPEZMARTíN, 2011, SÁEZ DE OCÁRIZ et al., 2014). En este sentido, las escuelas se convierten en espacios de convivencia espléndidos para educar actitudes constructivas, solidarias, responsables y equitativas, independientemente del genero de los participantes (GAVIRIA; CASTEJÓN, 2016, PÉREZ-GALLARDO et al., 2013).

La literatura científica constata que un clima escolar socialmente positivo influye tanto en el alumnado como en el profesorado en el desarrollo de actitudes basadas en el compromiso y respeto con los demás (e.g., GÓMEZ; MATAMALA; ALCOCEL, 2002). Por ello, las experiencias educativas que favorezcan la mejora de los estados de ánimo pueden contribuir positivamente a educar las relaciones interpersonales (REIGAL; VIDERA, 2013). De ahí el interés que los estudiantes universitarios en el ámbito de la actividad física y el deporte reconozcan la importancia de incidir sobre estas cuestiones en sus futuras intervenciones.

\section{COEDUCACIÓN Y CONVIVENCIA EN GRUPOS SEGREGADOS Y MIXTOS}

La coeducación se refiere al sistema pedagógico integral que promueve la igualdad de trato del alumnado de diferentes sexos que conviven en una realidad común (ALCALAY et al., 2000, VALDIVIA et al., 2012). Sin embargo, no se trata de simplemente mezclar el género masculino con el femenino, ni intentar que ambos hagan siempre lo mismo, sino de educar en el respeto mutuo en función de sus necesidades y del proyecto educativo elegido ( DÍAZAGUADO; MARTÍNEZ, 2011, MORENO et al., 2008, SUVERBIOLA, 2012)prestando especial atencilu00f3n a los estudios de la Unidad de Psicologlu00eda Preventiva (UCM. Educar la convivencia coeducativa implica desarrollar las bases del respeto, la libertad y la valoración de uno mismo y de los demás, independientemente del género (CASTILLA, 2008).

De hecho, existen estudios que afirman de la ineficacia de proponer siempre las actividades en grupos mixtos, puesto que lejos de favorecer la igualdad de trato pueden acentuar los estereotipos de género aún presentes en nuestra sociedad (CERVIÑO, 2007; SUVERBIOLA, 2012). Por ejemplo, se confirma que las chicas se implican menos que los chicos ante propuestas comunes; los chicos sienten vergüenza y demuestran una vaga motivación cuando se proponen experiencias relacionadas con actividades consideradas femeninas como la danza o la expresión corporal; y que a menudo monopolizan la atención y la comunicación interpersonal, coartando a las chicas (CANALES; REY, 2014, TORRENTS et al., 2011, VALDIVIA et al., 2013). 
También se pone de manifiesto, siempre desde propuestas coeducativas, el interés de ambos sexos por disponer de momentos de interacción con su mismo género para compartir intereses comunes, evitar las comparaciones y garantizar la seguridad y comodidad al desarrollar temáticas concretas en ambientes de libre expresión, respeto y comprensión entre iguales (ALCALAY et al., 2000, DÍAZ-AGUADO; MARTíNEZ, 2011, LYU; GILL, 2011, VILANOVA; SOLER, 2012).

Desde edades bien tempranas, nuestra sociedad occidental continua asociando la fuerza, la resistencia, la agresividad, el riesgo y las cualidades instrumentales al género masculino, mientras que las situaciones más tranquilas, relacionadas con el ritmo, la elasticidad, la flexibilidad, la coordinación y las capacidades expresivas se vinculan al género femenino (BLÁNDEZ; FERNÁNDEZ-GARCÍA; SIERRA, 2007, DEL CASTILLO; CORRAL, 2011, MORENO-MURCIA et al., 2011). Muestra de ello, se observa que en las sesiones de educación física las chicas prefieren las prácticas motrices cooperativas y expresivas, mientras que los chicos muestran preferencia por juegos y deportes de cooperación-oposición, sobre todo en los que existe competición (SÁENZ-LÓPEZ; SICILIA; MANZANO, 2010, SOLER, 2009).

Sin embargo, las desigualdades entre chicos y chicas no surgen tanto de los estereotipos asignados al tipo de actividad motriz sino de las interacciones entre participantes de ambos géneros que se generan durante el juego (SOLER, 2009, VALDIVIA et al., 2012). En consecuencia, la formación de los futuros formadores en temas de coeducación, es una pieza clave para la transformación de los procesos socio-educativos desde el reconocimiento de las características y necesidades de cada género (AZORÍN, 2014, GARCÍA et al., 2013).

La participación en ciertas actividades con personas del mismo género ofrece al alumnado la oportunidad de desarrollar en libertad conductas exitosas que a posteriori podrán compartir con personas del otro género, produciéndose una mejora de las relaciones interpersonales ( DEL CASTILLO et al., 2016, DÍAZ-AGUADO; MARTíNEZ, 2011).

De entre las estrategias pedagógicas para fomentar la coeducación (AZORÍN, 2014, DÍAZ-AGUADO; MARTÍNEZ, 2011, GARAIGORDOBIL, 2008), las actividades cooperativas son un recurso didáctico eficaz para favorecer actitudes positivas de convivencia entre chicos y chicas. Las relaciones positivas que genera resolver retos comunes de naturaleza cooperativa es una experiencia de interés para promover la interdependencia amistosa (GONZÁLEZ; GARCÉS; GARCÍA, 2012, LEÓN; GOZALO; POLO, 2012). Aprender a compartir experiencias positivas y cooperativas entre ambos géneros es un primer paso para comprender con empatía la necesidad de avanzar en favor de la igualdad de oportunidad para los hombres y las mujeres (DÍAZ-AGUADO; MARTÍNEZ, 2011).

\section{EDUCACIÓN FÍSICA Y EXPERIENCIAS POSITIVAS: LAS SITUACIONES MOTRICES DE EXPRESIÓN COOPERATIVAS}

La educación física es una disciplina basada en la acción motriz que favorece la adquisición y mejora de aprendizajes procedimentales, es decir, permite educar saberes asociados a resolver los problemas que plantea una situación motriz. Desde este área se puede promover un repertorio variado de experiencias motrices en función del tipo de relación motriz que interese activar (PARLEBAS, 2001). Asílas prácticas sociomotrices, caracterizadas por la presencia de compañeros 0 adversarios, movilizan una red de interacciones motrices entre los participantes ya sean chicas 
o chicos de gran interés para favorecer competencias sociales de convivencia (SÁEZ DE OCÁRIZ et al., 2014). Además si se trata de actividades cooperativas y no competitivas, como la expresión motriz, se está ante recursos extraordinarios para la promoción de conductas prosociales basadas en el respeto y la ayuda mutua (FERNÁNDEZ-CABEZAS et al., 2011, GREEN; RECHIS, 2006, LAVEGA et al., 2011, LAVEGA et al., 2014, SÁEZ DE OCÁRIZ et al., 2014).

Se trata de prácticas que buscan representar un mensaje simbólico mediante acciones motrices. Al realizarse en equipo las personas deben ponerse de acuerdo para compartir la doble función de actor y espectador. En efecto, en estas situaciones motrices siempre habrá unos actores que representan conjuntamente un proyecto expresivo; y al mismo tiempo unos espectadores que observan esa representación simbólica (MATEU, 2010).

Por todo ello, a través de la expresión motriz se pueden crear ambientes de estados deánimo positivos que favorezcan el bienestar personal y también la convivencia grupal (GARCÍA-RAGA; LÓPEZ-MARTÍN, 2011, GELPI et al., 2014, SÁEZ DE OCÁRIZ et al., 2014). Sin embargo, todavía existe un número reducido de estudios que aporte evidencias científicas sobre la contribución de este tipo de prácticas en la mejora de los estados de ánimo asociados a la convivencia con los demás (REIGAL et al., 2013, REIGAL; VIDERA, 2013, TORRENTS et al., 2011).

A partir de estas consideraciones la presente investigación se planteó como objetivo examinar el efecto de las situaciones motrices de expresión cooperativa (SMEC) sobre los estados de ánimo de estudiantes universitarios al participar en grupos segregados (chicoschicos, chicas-chicas) y en grupos mixtos (chicas-chicos).

\section{MÉTODO}

\subsection{Participantes}

Este estudio se llevó a cabo con 81 estudiantes de primer curso del grado en Ciencias de la Actividad Física y el Deporte, de la asignatura de expresión corporal y danza impartida en el Instituto Nacional de Educación Física de Cataluña de Barcelona, 22 mujeres (27.2\%) y 59 hombres (72.8\%) con un rango de edad de 18 a 27 años ( $M=21.05$ años, $D T=2.06)$. Todos los estudiantes dieron su consentimiento para participar voluntariamente en esta investigación, que fue aprobada por el comité de ética de la universidad (fecha, 23 de marzo de 2015, Comité Ético de Investigación Clínica (CEIC) núm. 1438).

\subsection{Instrumento}

Se empleó la versión reducida adaptada y validada al español del cuestionario POMS (ANDRADE et al., 2013). Se trata de uno de los cuestionarios más utilizados en psicología del deporte (LEUNES, 2000). Los numerosos estudios que se han llevado a cabo indican que se pueden identificar, medir de manera fiable los estados de ánimo en el colectivo de participantes seleccionados. Con el fin de adaptar el POMS al específico contexto de la educación, se han creado distintas versiones reducidas del mismo y como resultado de estas revisiones se ha optado por utilizar la escala propuesta por ANDRADE et al. (2013).

Esta escala identifica seis ámbitos de estados de ánimo: Puntuación Global (PG), Tensión-Ansiedad (TA), Depresión-Abatimiento (DA), Rabia-Hostilidad (RH), Fatiga-Inmovilidad $(\mathrm{FI})$, y Vigor-Actividad (VA). Los ítems se valoran de nada (0) a muchísimo (4). Según estos 
autores el factor Tensión-Ansiedad está relacionado con un aumento de la tensión músculoesquelética. La Depresión-Abatimiento representan un estado de depresión acompañado por una sensación de incapacidad personal. El factor Rabia-Hostilidad manifiesta un estado de rabia y antipatía dirigido hacia los demás. Las escalas de Vigor-Actividad corresponden a un estado de vigor, entusiasmo y un considerable aumento de energía. La Fatiga-Inmovilidad, representa un estado de cansancio generalizado, acompañado de inmovilidad y baja energía. Además se obtiene una puntuación global de estado de ánimo; dicha puntuación se obtiene sumando los valores de los cuatro factores que se relacionan directamente como son la Tensión-Ansiedad, Depresión-Abatimiento, Rabia-Hostilidad, Fatiga-Inmovilidad, y restando el Vigor-Actividad.

\subsection{Procedimiento}

Se realizaron tres sesiones de 90 minutos de duración, estando los alumnos distribuidos en grupos de 40 personas. En la primera de ellas se llevo a cabo una sesión formativa impartida por la profesora de la asignatura (psicóloga y con formación superior en artes escénicas) para explicar las condiciones de participación en el estudio y familiarizar a los participantes con el cuestionario POMS. Posteriormente se llevaron a cabo dos sesiones con cinco SMEC cada una (ver Cuadro 1); la primera realizada en grupos segregados y la segunda en grupos mixtos. En el transcurso de esas sesiones la docente se limitó a explicar las diez situaciones motrices expresivas. Solo intervino cuando hubo dudas sobre las reglas de las tareas a realizar, al gestionar el tiempo de la actividad y al organizar el material.

Cuadro 1 - Descripción de las situaciones motrices de expresión cooperativa del estudio.

\begin{tabular}{|l|l|}
\hline \multicolumn{1}{|c|}{$\begin{array}{c}\text { SITUACIÓN } \\
\text { MOTRIZ }\end{array}$} & \multicolumn{1}{c|}{ DESCRIPCIÓN } \\
\hline $\begin{array}{l}\text { Yo cóncavo y tú } \\
\text { convexo }\end{array}$ & $\begin{array}{l}\text { En parejas, uno se sitúa inmóvil en una posición que describa una curva, y la otra persona } \\
\text { 'rellena' con su cuerpo el espacio libre creado. Posteriormente se cambian de roles. }\end{array}$ \\
\hline $\begin{array}{l}\text { El árbol } \\
\text { colectivo }\end{array}$ & $\begin{array}{l}\text { En grupos de 5-6 personas se tumban unos sobre otros respirando con un ritmo común. } \\
\text { Progresivamente y en orden, los componentes se levantan bailando y se tumban en el } \\
\text { suelo manteniendo el contacto con el resto de componentes. }\end{array}$ \\
\hline Hielo y fuego & $\begin{array}{l}\text { Se divide al grupo en dos roles: hielo y fuego. El 'hielo' permanece inmóvil y el 'fuego' } \\
\text { pueden entablar un dialogo corporal con el 'hielo' para convertirlo en 'agua'. Así } \\
\text { sucesivamente. Posteriormente se cambian de roles. }\end{array}$ \\
\hline $\begin{array}{l}\text { Juntos no pero } \\
\text { si revueltos }\end{array}$ & $\begin{array}{l}\text { En parejas, se colocan uno enfrente del otro y representan una secuencia de figuras } \\
\text { diseñadas previamente por cada uno de los integrantes. }\end{array}$ \\
\hline Baile tribal & $\begin{array}{l}\text { En grupos se elabora una composición coreográfica donde cada persona aporta una } \\
\text { consigna de acción para entre todos ellos crear una 'frase musical'. }\end{array}$ \\
\hline $\begin{array}{l}\text { Máscara a } \\
\text { cuatro manos }\end{array}$ & $\begin{array}{l}\text { En parejas, dan forma a las cuatro manos y realizan una máscara sobre el rostro de uno/a } \\
\text { de ellos. Posteriormente se cambian de roles. }\end{array}$ \\
\hline Encajándonos & $\begin{array}{l}\text { Formar figuras grupales de cuatro a partir de la suma de figuras individuales que se } \\
\text { encajan, trasladándose de una figura grupal a otra con movimientos creativos improvisados. }\end{array}$ \\
\hline $\begin{array}{l}\text { Máximo y } \\
\text { mínimo }\end{array}$ & $\begin{array}{l}\text { En parejas y manteniendo alguna parte del cuerpo unida, investigar diferentes figuras } \\
\text { ocupando el máximo y mínimo espacio. }\end{array}$ \\
\hline $\begin{array}{l}\text { La pareja más } \\
\text { sonora }\end{array}$ & \begin{tabular}{l} 
En parejas, inventar un saludo sonoro a través del contacto con diversas partes del cuerpo. \\
\hline En grupos se elabora una coreografía grupal a través de música orgánica (sonido que \\
surge del cuerpo en contacto con la propia persona, los compañeros o el suelo, e implica \\
movimiento global).
\end{tabular} \\
\hline
\end{tabular}

Nota: En todas las situaciones se compartía el doble rol de actor y espectador.

Fuente: construcción propia de los investigadores 
El alumnado cumplimentó el cuestionario POMS al inicio y al final de cada una de las sesiones, siendo el equipo investigador el encargado de recoger todos los formularios al acabar la sesión. Los participantes indicaron en el cuestionario el valor que mejor se ajustaba a su estado de ánimo en ese preciso momento.

\subsection{Análisis de los datos}

Se estudió el efecto de la variable independiente género del grupo (grupo segregado, grupo mixto) sobre las variables dependientes, en concreto los seis factores de estado de ánimo (POMS): 1) $P G(P G=[T A+D A+R H+F I]-V A+100)$; 2) TA, 3) DA, 4) RH, 5) Fl, y 6) VI.

Al tratarse de variables correlacionadas no Normales (aspecto que se comprobó previamente), se optó por emplear el Modelo Lineal Generalizado, basado en Ecuaciones de Estimación Generalizadas, ajustando una distribución de la familia Gaussiana (GEE). Para realizar ese análisis se utilizó el software estadístico SPSS v.19.0.

\section{RESULTADOS}

Los resultados de la variable independiente sobre los diferentes estados de ánimo recogidos por el cuestionario POMS se presentan en función del comportamiento de dichos factores tras participar en tareas de SMEC tanto en grupos segregados (chicas-chicas, chicoschicos) como en grupos mixtos (chicas-chicos).

El análisis estadístico de los 609 registros $(n=81)$ reflejó diferencias significativas $(p<$ .05) en algunos de los factores estudiados (ver Tabla 1).

Tabla 1 - Significación de los efectos principales.

\begin{tabular}{|c|c|c|c|c|}
\hline \multirow{2}{*}{ Factor } & \multirow{2}{*}{ Origen } & \multicolumn{3}{|c|}{ Tipo III } \\
\hline & & $X^{2}$ de Wald & gl & $p$ \\
\hline Puntuación Global & Género Grupo & 7.78 & 1 & .005 \\
\hline Tensión-Ansiedad & Género Grupo & 7.51 & 1 & .006 \\
\hline Depresión-Abatimiento & Género Grupo & 20.46 & 1 & $<.001$ \\
\hline Rabia-Hostilidad & Género Grupo & 17.75 & 1 & $<.001$ \\
\hline Fatiga Inmovilidad & Género Grupo & 12.22 & 1 & $<.001$ \\
\hline Vigor-Actividad & Género Grupo & 16.75 & 1 & $<.001$ \\
\hline
\end{tabular}

Fuente: construcción propia de los investigadores

\subsection{Estados de ánimo en grupos segregados y mixtos}

Al comparar los estados de ánimo del inicio con el final de las sesiones de situaciones motrices de expresión motriz cooperativa (SMEMC) se observó que ambos grupos disminuyeron la puntuación global. Ese descenso fue significativamente inferior $(p=.023)$ en el grupo segregado $(M=-10.40, S D=3.50)$ respecto al grupo mixto $(M=-5.90, S D=2.82)$. (Ver Figura 1).

Tras actuar en SMEC los participantes en grupos segregados disminuyeron de manera significativa $(p<.001)$ los valores de tres estados negativos: Depresión-Abatimiento $(M=-0.09$, $S D=.63)$, Rabia-Hostilidad $(M=-0.17, S D=1.01)$, Fatiga-Inmovilidad $(M=-2.62, S D=0.99)$ 
y aumentaron el valor del estado positivo Vigor-Actividad $(M=2.89, S D=1.46)$. Sin embargo aumentó significativamente $(p<.001)$ la intensidad del valor Tensión-Ansiedad $(M=1.91, S D$ $=2.39$ ).

Después de intervenir en SMEC los participantes en grupos mixtos disminuyeron de manera significativa los valores de tres estados negativos: Tensión-Ansiedad $(M=-2.67, S D=$ 1.64), Depresión-Abatimiento $(M=-1.12, S D=.46)$, Fatiga-Inmovilidad $(M=-0.69, S D=.86)$ y aumentaron el valor del estado positivo Vigor-Actividad $(M=0.52, S D=1.3)$. Sin embargo también aumentaron la intensidad del valor Rabia-Hostilidad $(M=0.66, S D=0.87)$.

Los participantes en grupos mixtos disminuyeron más los valores de estados de ánimo negativos de Tensión-Ansiedad $(p=.005)$ y Depresión-Abatimiento $(p=.001)$ que los protagonistas en grupos segregados. La Tensión-Ansiedad $(M=-2.67, S D=1.64)$ fue el estado de ánimo negativo que alcanzó el valor inferior en el grupo mixto, mientras que en el grupo segregado fue el que registró el valor superior $(M=1.91, S D=2.39)$.

Los grupos segregados mostraron valores inferiores de Fatiga-Inmovilidad $(p=.001)$, así como valores superiores de Vigor-Actividad $(p=.005)$ respecto a los grupos mixtos. Asimismo, pese a no encontrarse diferencias significativas $(p>.05)$ respecto al grupo mixto los valores en Rabia-Hostilidad tendieron a ser inferiores.

Figura 1 - Comportamiento de las diferentes dimensiones en los grupos segregados y mixtos

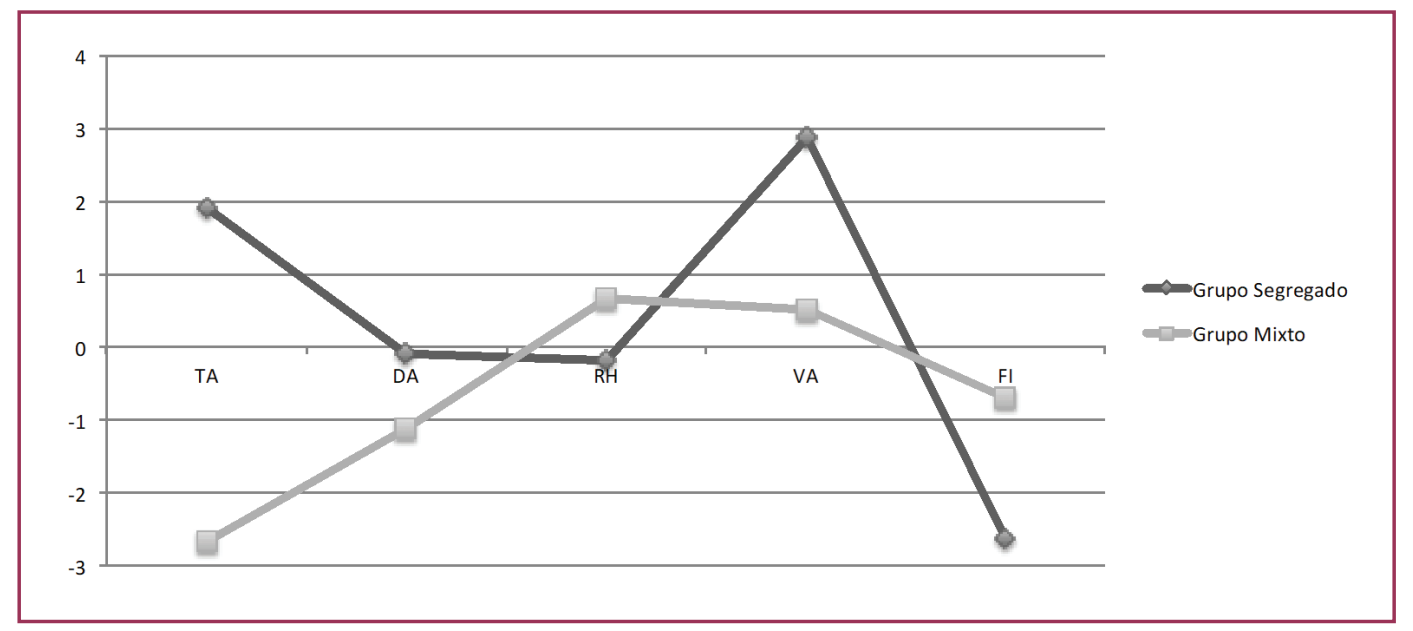

Nota: TA: tensión-ansiedad; DA: depresión-abatimiento; RH: rabia-hostilidad; VA: vigor-actividad; FI: fatiga-inmovilidad. Fuente: construcción propia de los investigadores

\section{DISCUSIÓN}

El presente estudio examinó el efecto de las situaciones motrices de expresión cooperativa (SMEC) sobre el estado de ánimo de los estudiantes universitarios que participaron en grupos segregados (chicos-chicos, chicas-chicas) y en grupos mixtos (chicos-chicas).

Los datos muestran que la participación en las SMEC mejora el estado anímico del alumnado al reducirse los valores de la puntuación global. Este escenario constata la aportación de dichas situaciones motrices a la creación de experiencias positivas que favorecen el bienestar personal y grupal de los alumnos (GARCÍA-RAGA; LÓPEZ-MARTíN, 2011, GELPI et al., 2014, LAVEGA et al., 2014, SÁEZ DE OCÁRIZ et al., 2014), tal y como se viene solicitando desde las 
directrices educativas actuales en España (ÁLVAREZ-GARCÍA et al., 2010, GARCÍA-RAGA; LÓPEZ-MARTÍN, 2011).

Asimismo, los resultados revelan que los efectos sobre los estados de ánimo de chicos y chicas, pese a ser positivos en ambos casos, son diferentes según el tipo de agrupación del alumnado. Se ha observado que los valores globales se modifican más favorablemente en el grupo segregado que en el mixto. Una posible interpretación puede estar asociada a la percepción cultural existente hacia la práctica de situaciones asociadas al género femenino, como es el caso de la expresión corporal (BLÁNDEZ et al., 2007, DEL CASTILLO; CORRAL, 2011, MORENO-MURCIA et al., 2011). Esa imagen femenina de estas prácticas podría provocar que tanto los chicos como las chicas afronten la experiencia de una manera más favorable al interaccionar con compañeros de su mismo género.

Estos hallazgos sugieren al profesorado de educación física la conveniencia de organizar a los alumnos mediante ambos tipos de agrupaciones, tanto para favorecer la convivencia entre ambos géneros en los grupos mixtos (CERVIÑO, 2007, SUVERBIOLA, 2012), como para ofrecer a los estudiantes la participación en ambientes de libre expresión, respeto y comprensión entre iguales, al permitirles participar en grupos segregados (ALCALAY et al., 2000, DÍAZ-AGUADO; MARTÍNEZ, 2011, LYU; GILL, 2011, VILANOVA; SOLER, 2012). Ambas posibilidades se complementan teniendo en cuenta las necesidades de cada género, produciéndose un incremento positivo de las relaciones interpersonales (DÍAZ-AGUADO; MARTÍNEZ, 2011, MORENO et al., 2008).

Al considerar el comportamiento de las diferentes dimensiones valoradas en los estados de ánimo del alumnado, los datos manifiestan que las actividades de expresión cooperativa originan en ambos grupos unos efectos positivos en la Depresión-Abatimiento, Vigor-Actividad y Fatiga-Inmovilidad.

Los resultados indican una reducción significativa en los valores de DepresiónAbatimiento, sobre todo en el grupo mixto, argumentados tanto por la propia naturaleza de las SMEC, que potencian conductas de respeto y ayuda mutua (FERNÁNDEZ-CABEZAS et al., 2011, GREEN; RECHIS, 2006, LAVEGA et al., 2011, SÁEZ DE OCÁRIZ et al., 2014), como por el hecho de compartir propuestas motrices en las que los roles de actor y espectador se combinan (MATEU, 2010). Dichas vivencias permiten al alumnado interaccionar de manera creativa y divertida con sus compañeros, creando ambientes positivos alejados de la depresión o abatamiento (GARCÍA-RAGA; LÓPEZ-MARTÍN, 2011, GELPI et al., 2014, SÁEZ DE OCÁRIZ et al., 2014).

Paralelamente, los datos revelan que aumentan significativativamente los valores de Vigor-Actividad, sobre todo en grupos segregados. Dichos hallazgos confirman el desarrollo de las interacciones interpersonales de los estudiantes en ambientes favorables (GONZÁLEZ et al., 2012, LEÓN et al., 2012), que fluyen al tener que crear mensajes simbólicos a través de proyectos expresivos conjuntos (MATEU, 2010). La participación con compañeros del mismo género permite desarrollar en libertad relaciones positivas interpersonales que repercuten positivamente en la mejora de la convivencia escolar (DÍAZ-AGUADO; MARTínEZ, 2011, GÓMEZ et al., 2002).

Asimismo, se observa que las SMEC disminuyen en ambos grupos los valores de Fatiga-Inmovilidad. La esencia de las SMEC, caracterizadas por el bajo coste energético y la 
ausencia de competición permiten a los estudiantes sentirse tranquilos y relajados al no tener que comparar sus resultados (SÁENZ-LÓPEZ et al., 2010).

Por otro lado, a pesar de los efectos positivos de las SMEC sobre el estado de ánimo, los datos confirman que las dimensiones Tensión-Ansiedad y Rabia-Hostilidad se comportan en sentido inverso en función del tipo de agrupación. En el grupo mixto los valores de TensiónAnsiedad se reducen significativamente y los de Rabia-Hostilidad sufren un aumento, mientras que en el grupo segregado se observa un incremento de los valores de Tensión-Ansiedad y una ligera disminución de los de Rabia-Hostilidad. Las diferentes aproximaciones de cada uno de los géneros hacia la puesta en escena y los sentimientos de vergüenza que afloran durante la práctica de las SMEC (CANALES; REY, 2014, TORRENTS et al., 2011, VALDIVIA et al., 2013), argumentan dichos resultados.

En este sentido, se interpreta que al participar conjuntamente chicos y chicas es posible reducir el nivel de tensión y ansiedad que provoca el nerviosismo escénico. En este caso, el "contagio emocional" (LAVEGA et al., 2011) de actuar con chicas que están más habituadas a este tipo de intervenciones puede ser un factor que ayude a reducir el estrés originado (BLÁNDEZ et al., 2007, DEL CASTILLO; CORRAL, 2011, MORENO-MURCIA et al., 2011, SÁENZ-LÓPEZ et al., 2010). Paralelamente, el miedo al ridículo manifestado por algunos alumnos al participar de las SMEC (CANALES; REY, 2014, TORRENTS et al., 2011, VALDIVIA et al., 2013), pueden explicar que el sentimiento de rabia se incremente al tener que realizar escenificaciones delante del género contrario, puesto que no se sienten con la suficiente libertad como para desarrollar conductas exitosas (DÍAZ-AGUADO; MARTíNEZ, 2011).

Una primera conclusión a la luz de los hallazgos descritos es la contribución eficaz que proporcionan las SMEC para favorecer en el contexto de la educación física experiencias coeducativas positivas significativas en el bienestar afectivo, subjetivo y social. Se trata de recursos que puede emplear el docente para facilitar experiencias motrices de interés para la educación de actitudes de respeto y compromiso hacia uno mismo y también hacia los demás (GARCÍA-RAGA; LÓPEZ-MARTÍN, 2011, GÓMEZ et al., 2002, SÁEZ DE OCÁRIZ et al., 2014).

Asimismo, queda confirmado que las SMEC pueden transformar de modo positivo los estados de ánimo en un contexto educativo que considere la coeducación y la equidad de género, respetando sus intereses y necesidades (AZORÍN, 2014, DÍAZ-AGUADO; MARTÍNEZ, 2011, GARAIGORDOBIL, 2008, GELPI et al., 2014, PARLEBAS, 2001). Se convierten en un instrumento docente coeducativo eficaz para el desarrollo de la convivencia escolar y las relaciones interpersonales en ambos géneros, tanto en grupos segregados como en grupos mixtos, en función de proyecto pedagógico que se quiera promover.

No obstante se reconoce la necesidad de continuar el estudio con otros tipos de participantes con el objetivo de profundizar en las posibilidades que ofrecen las SMEC al profesorado de educación física en la búsqueda de recursos docentes coeducativos para mejorar la convivencia escolar. 


\section{REFERENCIAS}

ALCALAY, Lidia et al. ¿Coeducación o Educación Segregada por Sexo? Una aproximación desde la perspectiva de la educación de género. Psykhe, v. 9, n. 2, p.171-179, 2000.

ÁLVAREZ-GARCÍA, David et al. La formación de los futuros docentes frente a la violencia escolar. Revista de Psicodidáctica, v. 15, n. 1, p. 35-56, 2010.

ANDRADE, Elena et al. Versión breve en español del cuestionario POMS para deportistas adultos y población general. Revista de Psicología del Deporte, v. 22, n. 1, p. 95-102, 2013.

ARNAIZ, Pilar. Escuelas eficaces e inclusivas: cómo favorecer su desarrollo. Educatio Siglo XXI, v. 30, n. 1, p. 25-44, 2012.

AZORÍN, Cecilia. Actitudes del profesorado hacia la coeducación: claves para una educación inclusiva. Ensayos, Revista de la Facultad de Educación de Albacete, v. 29, n. 2, p. 159174, 2014.

BLÁNDEZ, Julia; FERNÁNDEZ-GARCÍA, Emilia; SIERRA, Miguel Ángel. Estereotipos de género, actividad física y escuela: La perspectiva del alumnado. Profesorado, Revista de Curriculum y Formación del Profesorado, v. 11, n. 2, p. 1-21, 2007.

CANALES, Inma; REY, Ana. Diferencias de género percibidas por el alumnado en la interacción visual y táctil de las tareas de expresión corporal. Movimento, v. 20, n. 1, p. 169-192, 2014.

CASTILLA, Ana Belén. Coeducación: pautas para su desarrollo en los centros educativos. Escuela Abierta, v. 11, p. 49-85, 2008.

CERVIÑO, María Jesús. ¿Qué es coeducación? Madrid: CEAPA, 2007.

DEL CASTILLO, Oscar et al. Estudio observacional del sexismo en la educación física del sur de España. Movimento, v. 22, n. 1, p. 263-278, 2016.

DEL CASTILLO, Oscar; CORRAL, Juan Antonio. El profesorado frente a la discriminación de género: uso de la retroalimentación. Cultura y Educación, v. 23, n. 4, p. 487-498, 2011.

DÍAZ-AGUADO, María José; MARTÍNEZ, Gema. Convivencia y aprendizaje escolar en la adolescencia desde una perspectiva de género. Psicothema, v. 23, n. 2, p. 252-259, 2011.

FERNÁNDEZ-CABEZAS, María et al. Desarrollo de la competencia social y prevención del comportamiento antisocial en niños de 3 años. Infancia y Aprendizaje, v. 34, n. 3, p. 337-347, 2011.

GARAIGORDOBIL, Maite. Evaluación de los efectos de un programa de juego cooperativo para niños de 10-11 años en la adaptación social y en la percepción que padres, profesores y compañeros tienen de las conductas pro-sociales de los niños. Infancia y Aprendizaje, v. 31, n. 3, p. 303-318, 2008.

GARCÍA, Rafael et al. Formación inicial del profesorado sobre género y coeducación: impactos metacognitivos de la inclusión curricular transversal sobre sexismo y homofobia. Profesorado, Revista de Curriculum y Formación del Profesorado, v. 17, n. 1, p. 269-287, 2013.

GARCÍA-PERALES, Ramón. La educación desde la perspectiva de género. Ensayos, Revista de la Facultad de Educación de Albacete, v. 27, p. 1-18, 2012. 
GARCÍA-RAGA, Laura; LÓPEZ-MARTÍN, Ramón. Convivir en la escuela. Una propuesta para su aprendizaje por competencias. Revista de Educación, v. 356, p. 531-555, 2011.

GAVIRIA, Didier; CASTEJÓN, Francisco. Desarrollo de valores y actitudes a través de la clase de educación física. Movimento, v. 22, n. 1, p. 251-262, 2016.

GELPI, Paula et al. La educación emocional a través de las prácticas motrices de expresión. Perspectiva de género. Educatio Siglo XXI, v. 32, n. 2, p. 49-70, 2014.

GIL-MADRONA, Pedro et al. Las niñas también quieren jugar: la participación conjunta de niños y niñas en actividades físicas no organizadas en el contexto escolar. Movimento, v. 20, n. 1, p. 103-124, 2014.

GÓMEZ, Concepción; MATAMALA, Rosa; ALCOCEL, Teresa. La convivencia escolar como factor de calidad. Revista Interuniversitaria de Formación de Profesorado, v. 5, n. 1, p. 1-6, 2002.

GONZÁLEZ, Juán; GARCÉS, Enrique; GARCÍA, Ana. Indicadores de bienestar psicológico percibido en alumnos de educación física. Revista de Psicología del Deporte, v. 21, n. 1, p. 183-187, 2012.

GREEN, Vanessa; RECHIS, Ruth. Children's cooperative and competitive interactions in limited resource situations: A literature review. Applied Developmental Psychology, v. 27, p. 42-59, 2006.

LAVEGA, Pere et al. Understanding emotions through games: Helping trainee teachers to make decisions. Electronic Journal of Research of Educational Psychology, v. 9, n. 2, p. 617-640, 2011.

LAVEGA, Pere et al. Efecto de la cooperación motriz en la vivencia emocional positiva: perspectiva de género. Movimento, v. 20, n. 2, p. 593-618, 2014.

LEÓN, Benito; GOZALO, Margarita; POLO, María Isabel. Aprendizaje cooperativo y acoso entre iguales. Infancia y Aprendizaje, v. 35, n. 1, p. 23-35, 2012.

LEUNES, Arnold. Updated bibliography on the profile of mood states in sport and exercises psychology research. Journal of Applied Sport Psychology, v. 12, n.1, p. 110-113, 2000.

LYU, Minjeong; GILL, Diane. Perceived physical competence, enjoyment and effort in same匹sex and coeducational physical education classes. Educational Psychology, v. 31, n. 2, p. 247260, 2011.

MATEU, Mercé. Observación y análisis de la expresión motriz escénica. Estudio de la lógica interna de los espectáculos artísticos profesionales: Cirque du Soleil (1986-2005). (Tesis inédita). Universidad de Barcelona, Barcelona, 2010.

MORENO, Juán Antonio et al. Coeducación y climas de aprendizaje en educación física. Aportaciones desde la teoría de Metas de Logro. Revista Internacional de Ciencias del Deporte, v. 11, n. 4, p. 42-64, 2008.

MORENO-MURCIA, Juán Antonio et al. Relación de los motivos de práctica deportiva en adolescentes con la percepción de competencia. Cultura y Educación, v. 23, n. 4, p. 533-542, 2011.

PARLEBAS, Pierre. Juegos, deporte y sociedad: Léxico de Praxiología Motriz. Barcelona: Paidotribo, 2001. 
PÉREZ-GALLARDO, Eloy et al. El dialogo como instrumento para la resolución de conflictos en escolares de educación secundaria. International Journal of Developmental and Educational Psychology, v. 1, n. 2. p. 189-194, 2013.

REIGAL, Rafael et al. Efecto agudo de la actividad fisicodeportiva y la expresión corporal sobre el estado de ánimo. Apunts. Educacion Fisica y Deportes, v. 113, n. 3, p. 30-36, 2013.

REIGAL, Rafael; VIDERA, Antonio. Efectos de una sesión de actividad física sobre el estado de ánimo. Revista Internacional de Medicina y Ciencias de la Actividad Física y el Deporte, v. 13, n. 52, p. 783-798, 2013.

SÁENZ-LÓPEZ, Pedro; SICILIA, Álvaro; MANZANO, José Ignacio. La opinión del profesorado sobre la enseñanza de la educación física en función del género. Revista Internacional de Medicina y Ciencias de la Actividad Física y el Deporte, v. 10, n. 37, p. 167-180, 2010.

SÁEZ DE OCÁRIZ, Unai et al. Emociones positivas y educación de la convivencia escolar. Contribuciones de la expresión motriz cooperativa. Revista de Investigación Educativa, v. 32, n. 2, p. 309-326, 2014.

SOLER, Susanna. Los procesos de reproducción, resistencia y cambio de las relaciones tradicionales de género en la educación física: el caso del fútbol. Cultura y Educación, v. 21, n. 1, p. 31-42, 2009.

SUVERBIOLA, Iratxe. Coeducación: un derecho y un deber del profesorado. Revista Electrónica Interuniversitaria de Formación del Profesorado, v. 15, n. 3, p. 59-67, 2012.

TORRENTS, Carlota et al. Posibilidades de las tareas de expresión corporal para suscitar emociones en el alumnado. Revista de Psicología del Deporte, v. 20, n. 2, p. 401-412, 2011.

UNESCO. Atlas Mundial de la Igualad de Género en la Educación. Paris: UNESCO, 2012.

VALDIVIA, Pedro et al. La experiencia docente en coeducación del profesorado de Educación Física. Profesorado, Revista de Curriculum y Formación del Profesorado, v. 17, n. 2, p. 385-399, 2013.

VALDIVIA, Pedro et al. Concepto de coeducación en el profesorado de educación Física y metodología utilizada para su trabajo. Movimento, v. 18, n. 4, p. 197-217, 2012.

VILANOVA, Anna; SOLER, Susanna. La coeducación en la educación física en el siglo XXI: reflexiones y acciones. Tandem, v. 40, p. 75-83, 2012. 